\title{
Theta burst stimulation influence the expression of BDNF in the spinal cord on the experimental autoimmune encephalomyelitis
}

 \\ Milica Ninkovic ${ }^{1}$ \\ ${ }^{1}$ Institute of Medical Research, Military Medical Academy, Belgrade, Serbia, ${ }^{2}$ Medical Faculty of Military Medical Academy, \\ University of Defence, Serbia, ${ }^{3}$ Center of Veterinary Services, Ministry of Defence, Serbia, ${ }^{4}$ Institute for Biological Research "Sinisa \\ Stankovic", University of Belgrade, Serbia, ${ }^{5}$ Institute for Biochemistry, Faculty of Medicine, University of Nis, Serbia
}

\begin{abstract}
Repetitive transcranial magnetic stimulation (rTMS) induces changes in expression of proteins engaged in the activity of excitatory and inhibitory systems, restores these functions and suppresses the progression of disability in experimental autoimmune encephalitis (EAE). The structural type of TMS, the arrangement as theta burst stimulation (TBS) has been applied as intermittent TBS (ITBS) and continuous TBS (CTBS) protocols to female adult DA rats. The animals were randomly divided into experimental groups: control group (C), group treated with complete Freund's adjuvant (CFA), experimental autoimmune encephalomyelitis (EAE) group, group treated with iTBS post EAE immunization (EAE + iTBS), group treated with cTBS post EAE immunization (EAE + CTBS), group of healthy animals treated with iTBS or CTBS. Therapeutic protocols of ITBS or CTBS in all EAE groups of animals were performed starting from 14 days post immunization (dpi), for 10 days with time point decapitation at 24 dpi. After decapitation, spinal cords were analysed for BDNF and Ki67 expression. The results revealed reduced BDNF expression in the rat's spinal cord of EAE animals in the stage of remission, which was associated with increased Ki67 and GFAP expressions. Decreased Iba 1 and BDNF expression, contrary to increased Iba 1 and Ki67 expression, suggests clustered microglia in the resolution phase of EAE. Enhanced GABA expression in spinal cord sections indicates higher GABA metabolic turnover, and also GAD activity in astrocytes, or prominent activity of GABAergic neurons. Both TBS protocols induced advance BDNF expression; amongst iTBS application provoked elevating of BDNF and stabilizing of GFAP and Ki67 expressions.
\end{abstract}

Key words: experimental autoimmune encephalomyelitis (EAE), spinal cord, TMS, BDNF.

\section{Introduction}

Major pathological processes in the central nervous system (CNS) during multiple sclerosis (MS) are neuronal inflammation and neurodegeneration [31]. Developing techniques directed to protect neuron structure and function; decreasing perivascular inflammation and supporting blood/brain barrier (BBB) integrity are promising in MS analogous CNS disease, i.e. experimental autoimmune encephalomyelitis (EAE).

According to present knowledge of factors influencing neuronal survival and repair during EAE, it has been shown that the proposed mechanism could be lymphoid imported and surrounding cells derived production of brain-derived neurotrophic factor (BDNF). Previously known as dorsal root gan- 
glion-derived, BDNF is known to enhance the neuronal survival following CNS damage, to promote the growth of dendrites and axons, and the synapse formation. It is a major factor of the neurogenesis, the migration, and differentiation of neurons [5]. Besides cytokines, BDNF is also a paracrine and autocrine signal molecule that interacts with specific receptors expressed on different cell types in the spinal cord. However, important aspects of its production in CNS auto-immunity such as cellular distribution and regulation remain unknown. Such data are important for the interpretation of the role of autoreactivity/inflammation in nonspecific CNS impairments, e.g. trauma or stroke, as well as for the evaluation of immunotherapy in MS.

The role of BDNF in EAE is controversial. Published data of mRNA in the lumbar spinal cord of EAE rats have shown that BDNF begins to upregulate 8 days post immunization (dpi), with a peak level at $12 \mathrm{dpi}$ and normalized expression at $16 \mathrm{dpi}$. The course of changes in BDNF is similar to changes in tumor necrosis factor $\alpha$ (TNF- $\alpha$ ) and interferon $\gamma$ (IFN- $\gamma$ ) [30]. Other data have shown that BDNF protein expression in the spinal cord was significantly increased at $12 \mathrm{dpi}$, and maintained at $15 \mathrm{dpi}$ with no significant change in mRNA levels [45].

Recent studies revealed that an external magnetic field, which is a consequence of transcranial magnetic stimulation (TMS) may affect BDNF content in the serum and cerebrospinal fluid (CSF). The results obtained from assessments of serum BDNF levels after TMS sessions are unclear. Some of them have found that repetitive TMS (rTMS) increases serum levels of BDNF, while the other studies have found no effect $[11,44]$. Wang et al. reported that high-frequency rTMS increases serum BDNF levels and the affinity of BDNF for TrkB receptors, whereas low-frequency TMS reduces BDNF levels [42].

Alternating magnetic fields during rTMS stimulate cortical neurons spreading the effects spatially and temporarily. Neuronal activity similar to longterm potentiation (LTP) and long-term depression (LTD) could support and create a favourable cellular environment for instigated neuroplasticity. Performing the activity in cortical zones it is forward to the lumbar spinal cord via corticospinal pathway activity. According to previously reported data, neuroplasticity similar to LTP might be induced by intermittent theta burst stimulation (iTBS) while continuous theta burst stimulation (cTBS) seems to induce the pattern of activity like LTD $[4,10]$. Neural plasticity induction with a BDNF released from surrounding cells could support growth and survival of neurons suffering from inflammatory/mediated oedema and energy deficiency as well as free radical attack from the neuroinflammation-activated astrocyte/microglia/lymphocyte [27]. Increased levels of BDNF within the dorsal root ganglion and spinal cord in EAE rats may facilitate myelin repair and remodel the synapses in the CNS. Anterograde transport of BDNF protein from the dorsal root ganglion to the dorsal horn of the spinal cord may promote neuroprotection.

The importance of BDNF is accentuated by interconnections of BDNF and $\gamma$-aminobutyric acid (GABA) availability. It has been reported that the progressive failure of neuronal connectivity, particularly transcallosal during MS development may be a result of impaired neuroinhibition [28]. Manyam et al. reported that the levels of GABA in the cerebrospinal fluid of patients with MS are significantly lower compared to healthy subjects [26]. Such data could be defended by the accepted facts that GABAergic neurons in the neocortex are parts of neural circuits involved in behaviour, perception, memory, and consciousness. In MS patients as well as EAE animals, most of these functions have been getting worse $[6,13,20,34,46]$.

Assuming that the release of BDNF in neural tissue is stimulated by rTMS, such protocol could be promising for the therapy of MS. Neuroplasticity induced by LTP and LTD may induce the local brain changes at the molecular level, such as higher availability of BDNF that may decrease inflammation and support myelin repair. The target of the study was to analyse both iTBS or CTBS-induced changes of BDNF expression in lumbar spinal cords of EAE rats, to establish whether synthesized BDNF is originated from astrocytes or microglia and to correlate it with GABA availability due to the TMS protocol used.

\section{Material and methods}

All experimental procedures were performed according to decision No. 323-07-00622/2017-05 obtained from the Ethics Committee for Animal Welfare and the Ministry of Agriculture and Environmental Protection of the Republic of Serbia, which respects the rules of the European Parliament's Directive 2010/63/EU on the protection of animals used for scientific purposes. 
Experiments were conducted on female Dark Agouti (DA) rats aged 10-14 weeks (150-200 g). The rats were housed in cages under standardized housing conditions (ambient temperature of $23 \pm 2^{\circ} \mathrm{C}$, relative humidity of $55 \pm 3 \%$ and a light/dark cycle of $13 \mathrm{~h} / 11 \mathrm{~h}$ ) with laboratory chow and water ad libitum. All the experiments were performed after 7 days' period of adaptation to laboratory conditions and were carried out between 9 a.m. and 1 p.m. During the period of paralysis, for the purpose of welfare, water and food were down positioned to rats or given manually.

The EAE was induced by a subcutaneous (s.c.) injection of $0.1 \mathrm{ml}$ solution of rat spinal cord tissue homogenate $(50 \% \mathrm{w} / \mathrm{v}$ in saline) suspended in complete Freund's adjuvant (CFA) containing $1 \mathrm{mg} / \mathrm{ml}$ Mycobacterium tuberculosis (CFA; Sigma, St. Louis, MO, USA). The suspension was injected in animal's right hind footpads. From the day of immunization, the rats were daily monitored and clinically scored.

Theta burst stimulation was performed using a MagStim Rapid2 device and a $25 \mathrm{~mm}$ figure-ofeight coil (The MagStim Company, Whitland, Dyfed, UK). The procedures of rTMS were done according to Huang et al. [17]. The iTBS protocol consisted of 20 trains of ten bursts ( 3 pulses at a frequency of $50 \mathrm{~Hz}$ ) repeated at $5 \mathrm{~Hz}$ (lasting $192 \mathrm{~s}$ with $10 \mathrm{~s}$ intervals between trains) (iTBS groups), while the cTBS protocol consisted of a single $40 \mathrm{~s}$ train of bursts repeated at $5 \mathrm{~Hz}$ (cTBS groups). Each block contained 600 pulses. Stimulus strength was $30 \%$ of the maximal stimulator output, just below the motor threshold value, which had been estimated as the visible contraction of upper limbs in all TBS-treated animals. During the TBS, the rats were slightly stressed and immobilized manually. The coil was gently relied on the rats' head, centring the coil right above bregma [25].

\section{Experimental procedure}

The animals were randomly divided into experimental groups: control group (C, $n=8)$; group treated with complete Freund's adjuvant (CFA, $n=8$ ), experimental autoimmune encephalomyelitis group (EAE, $n=8$ ), group treated with iTBS post EAE immunization (EAE + iTBS, $n=8$ ), group treated with cTBS post EAE immunization (EAE + CTBS, $n=8$ ), group of healthy animals treated with iTBS (iTBS, $n=8$ ) or cTBS (cTBS, $n=8$ ). Therapeutic protocols with iTBS or cTBS in all EAE groups were performed starting from $14 \mathrm{dpi}$, for 10 days with time point decapitation at $24 \mathrm{dpi}$. Healthy animals were treated with iTBS or cTBS for 10 days.

Animals before the immunization as well as all animals before the decapitation were intraperitoneally anaesthetized (sodium-pentobarbital $45 \mathrm{mg} / \mathrm{kg}$ body weight - b.w.). The decapitations were performed $24 \mathrm{~h}$ after the last TBS protocol or at the appropriate time point. Clinical evaluation of EAE was undertaken daily in a double-blind manner for up to 24 dpi [14]. All immunized animals developed clinical signs of the EAE that appeared between 10 and $12 \mathrm{dpi}$ and reached a peak at 11-14 dpi. Development of the EAE signs was accompanied by body weight loss in all experimental groups and maximal weight loss coincided with paralysis. Two different TBS protocols, which had been reported to modulate cortical excitability in opposite directions, induced similar effects on the clinical score in EAE-induced animals. Duration of paralysis was notably shorter in EAE animals by $49 \%$ (iTBS) and $54 \%$ (cTBS) treatment $(p<0.05$, respectively), compared to the EAE group.

\section{Immunofluorescence}

The experimental procedure for immunofluorescence staining was performed according to Dalmau and colleagues [7]. Spinal cords of each of three rats per group removed quickly and fixed in $4 \%$ paraformaldehyde (TAAB Laboratory Equipment, Aldermaston, UK) in $0.1 \mathrm{M}$ phosphate buffer (PBS; pH 7.4) for $12 \mathrm{~h}$ at $4^{\circ} \mathrm{C}$. For cryoprotection, lumbar spinal cord tissue was transferred into the graded sucrose (10-30\% in 0.1 M PBS, pH 7.4), frozen in 2-methyl butane, and kept at $-80^{\circ} \mathrm{C}$ until sectioning on a cryotome (CRIOCUT-E Reichert-Yung).

Sections of the spinal cord ( $25 \mu \mathrm{m}$ thick) were collected serially, mounted on superfrost glass slides, dried for $2 \mathrm{~h}$ at room temperature and stored at $-20^{\circ} \mathrm{C}$ until staining. Initially, one series of slides of all experimental groups was incubated with primary polyclonal rabbit anti-BDNF antibody (1:50; Thermo Fisher Scientific, USA) overnight at $4^{\circ} \mathrm{C}$, then secondary goat anti-rabbit Alexa Fluor 488 (1: 500; Invitrogen, Carlsbad, USA) for $2 \mathrm{~h}$ at room temperature in the dark. Subsequently, the sections were incubated with primary monoclonal mouse anti-GFAP (1 : 200; Elabscience, USA) or mouse monoclonal anti-Iba 1 (1: 50; Abcam, Germany) and 
after that with secondary donkey anti-mouse Alexa Fluor 555 (1:250; Invitrogen, Carlsbad, USA) for $2 \mathrm{~h}$ at room temperature in the dark.

The next series of spinal cord sections was incubated also with primary polyclonal rabbit anti-BDNF antibody (1: 50; Thermo Fisher Scientific, USA) overnight at $4^{\circ} \mathrm{C}$, but later with secondary donkey anti-rabbit Alexa Fluor 647 (1: 500; Abcam, Germany) for $2 \mathrm{~h}$ at room temperature in the dark. Afterwards, spinal cord sections were incubated with primary polyclonal guinea pig anti-GABA (1: 1000; Abcam, Germany) overnight at $4^{\circ} \mathrm{C}$ and then with secondary goat anti-guinea pig Alexa Fluor 488 ( 1 : 500; Invitrogen, Carlsbad, USA) for $2 \mathrm{~h}$ at room temperature in the dark.

A new series of slides was incubated with primary monoclonal rabbit anti-Ki67 antibody $(1: 300$; Thermo Fisher Scientific, USA) overnight at $4^{\circ} \mathrm{C}$, and later with secondary goat anti-rabbit Alexa Fluor 488 (1: 500; Invitrogen, Carlsbad, USA) for $2 \mathrm{~h}$ at room temperature in the dark. Afterward, slides were incubated with primary mouse monoclonal antiGFAP (1 : 200; Elabscience, USA) or mouse monoclonal anti-Iba 1 (1:50; Abcam, Germany) and then with secondary donkey anti-mouse Alexa Fluor 555 ( 1 : 250; Invitrogen, Carlsbad, USA) for $2 \mathrm{~h}$ at room temperature in the dark.

Finally, all slides were prepared on microscopic slides using the Mowiol medium (Sigma Aldrich) and analysed under the confocal microscope (Zeiss Axiovert 200M, LSM 510 laser module).

\section{Image processing and calculation of a colocalization coefficient}

Three sections of three animals per group were used to verify fluorescence intensity. From each section, five random photomicrographs in the ventral horn regions of the spinal cord were captured at magnification (40x objective). Semi-quantitative fluorescence intensity analysis was made using ImageJ software. Intensities were expressed as a mean \pm standard deviation (SD) of average grey values [41].

Quantitative colocalization analysis employs specialized algorithms which calculate a number of respective coefficients, according to which colocalization can be evaluated quantitatively [1]. As raw confocal images have high levels of background, they should be prepared to become suitable for reliable calculation of colocalization coefficients by correcting it. Colocalization is detected by correspondent antibodies with diverse excitation spectra when staining of antigens visualized in different colours overlaps.

Confocal microscopes provide resolution required for viewing colocalization in images clearly. This analysis can be explained as an existence of the signal at the same pixel location when examining multi-channel fluorescence microscopy images. The channels are generated by two different fluorochromes when respective antigens are visualized during examining of the same sample region. Observed colocalization of the antigens of interest gave valuable clues regarding their structural and functional characteristics.

Colocalization presented as a plate of three images consisting of fluorescence for red and green channels, as well as a third merged image where the channels are combined (overlapping pixels turned yellow). The analysis is assisted by computer software, which estimates the degree of colocalization according to specialized algorithms within the selected region of interest (ROI). The analysis is performed for the evaluation of the colour components of the selected pair of channels. Images were imported into the Fiji version of the free image processing software ImageJ. Fiji contains a number of pre-installed plugins including a procedure for colocalization analysis, designated as coloc2, which calculates a variety of colocalization parameters such as the Pearson coefficient and Manders coefficients, both of which are based on pixel-intensity-correlation measurements. Numerical correlation parameters are recorded, as well as the 2D intensity histogram for visualization of the correlation between the two channels. Threshold objects are binarized followed by a watershed segmentation to separate adjacent entities. The results of coloc2 derived intensity-based correlation analysis were copied into the same MS-Excel sheet and the different parameters, such as the Pearson coefficient or the Manders coefficients were multiplied with the colocalization fraction value. This results in a reduction of the pixel intensity-based correlation according to the percentage of object-based colocalization.

\section{Statistical analysis}

Statistical data analysis was performed using one-way ANOVA and Tukey's post hoc multiple tests (software GraphPad Prism, version 5.03). Values are presented as the means \pm SD. Differences were considered statistically significant for $p<0.05$. 


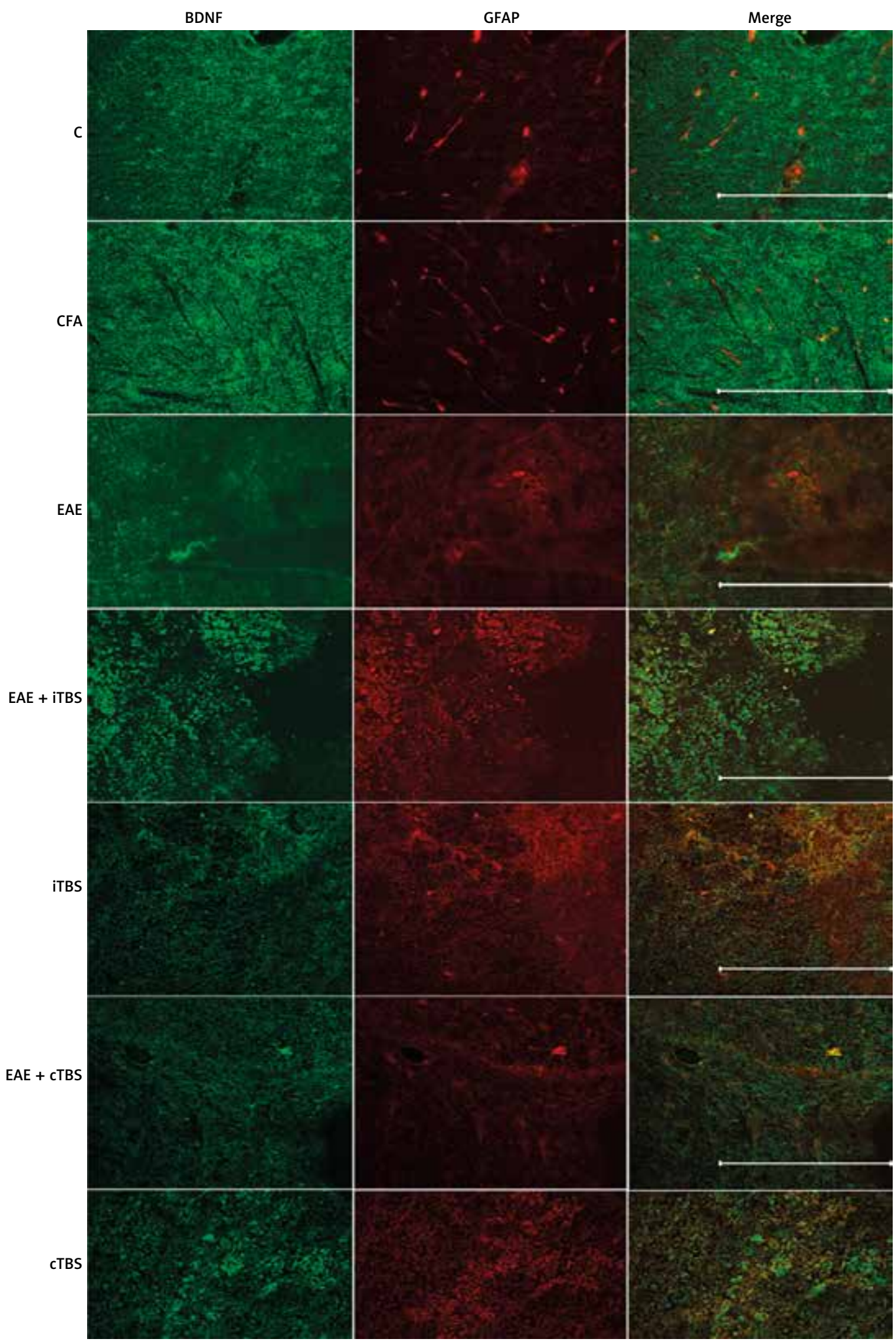

Fig. 1. Double fluorescence staining showing immunoreactivity for BDNF and GFAP in rats' spinal cords. Bright grey (green in a colour photograph) represents BDNF positivity, and darker grey (red in a colour photograph) represents GFAP positivity. C - control group, EAE - rats (EAE) with experimental autoimmune encephalomyelitis, CFA - rats treated with complete Freund's adjuvant, iTBS treatment on EAE animals (EAE + iTBS) or on healthy animals (iTBS) and cTBS treatment on EAE animals (EAE + CTBS) or on healthy animals (cTBS). The photomicrographs were taken at magnification 400x. Scale bar corresponds to $200 \mu \mathrm{m}$. 



Fig. 2. Effects of iTBS and CTBS on BDNF (A) and GFAP (B) expression in the spinal cords. Results were expressed as average grey value \pm SD $(n=3)$. Changes of Pearson's correlation coefficient $(C)$ and Manders coefficients (D): M1 (BDNF) and M2 (GFAP). Values are presented as means \pm SD. Differences were considered statistically significant for ${ }^{*} p<0.05$ compared to controls and $p<0.05$ compared to the EAE group.

\section{Results}

Photomicrographs of fluorescent immunoreactivity of BDNF and GFAP in the spinal cord tissue are shown in Figure 1, while analysed results are shown in Figure 2.

Decreased BDNF immunoreactivity was measured in EAE, but also in healthy animals treated with iTBS (Fig. 2A, $p<0.05$ ). Application of iTBS or cTBS on EAE animals induced BDNF elevation, compared to the EAE group $(p<0.05)$. A significant increase in GFAP was noted in the EAE group, compared to controls $(p<0.05)$, while cTBS treatment suppressed GFAP immunoreactivity, compared to the EAE group
(Fig. 2B, $p<0.05$ ). High positive coefficients of correlations of BDNF and GFAP were detected in both ITBS/CTBS groups with EAE and these correlations were significantly higher compared to control groups as well as compared to the EAE group (Fig. 2C, $p<0.05)$. Both Manders coefficients M1 (BDNF) and M2 (GFAP) increased in EAE + iTBS compared to both control and EAE groups (Fig. 2D, $p<0.05$, respectively). Although neither BDNF nor GFAP immunoreactivity in healthy animals treated with cTBS changed compared to controls, the correlation coefficient and Manders coefficient of colocalization notably increased $(p<0.05)$. 


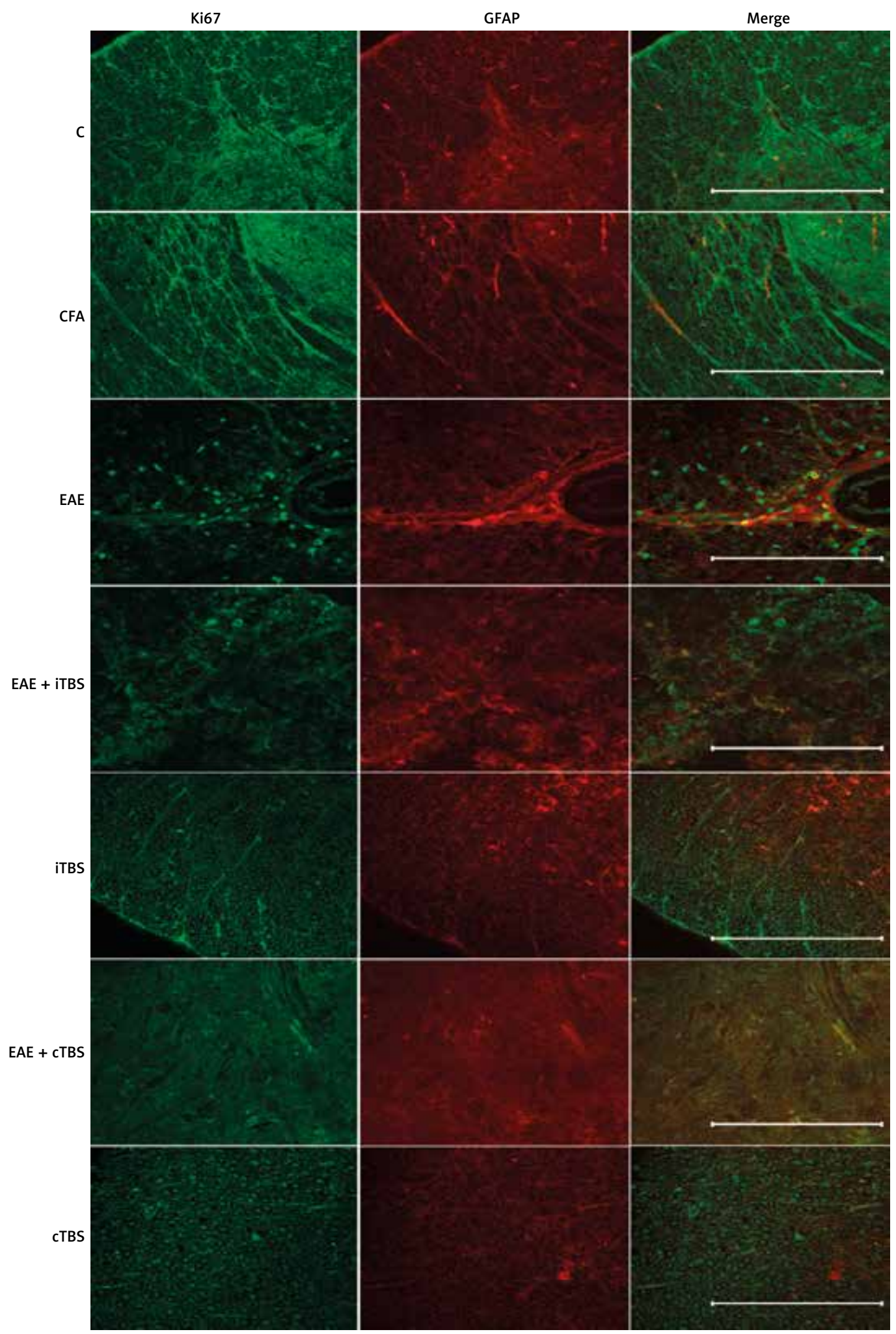

Fig. 3. Double fluorescence staining showing immunoreactivity for Ki67 and GFAP in rats' spinal cords. Bright grey (green in a colour photograph) represents Ki67 positivity, and darker grey (red in a colour photograph) represents GFAP positivity. C - control group, EAE - rats (EAE) with experimental autoimmune encephalomyelitis, CFA - rats treated with complete Freund's adjuvant, iTBS treatment on EAE animals (EAE + iTBS) or on healthy animals (iTBS) and cTBS treatment on EAE animals (EAE + CTBS) or on healthy animals (cTBS). The photomicrographs were taken at magnification 400x. Scale bar corresponds to $200 \mu \mathrm{m}$. 

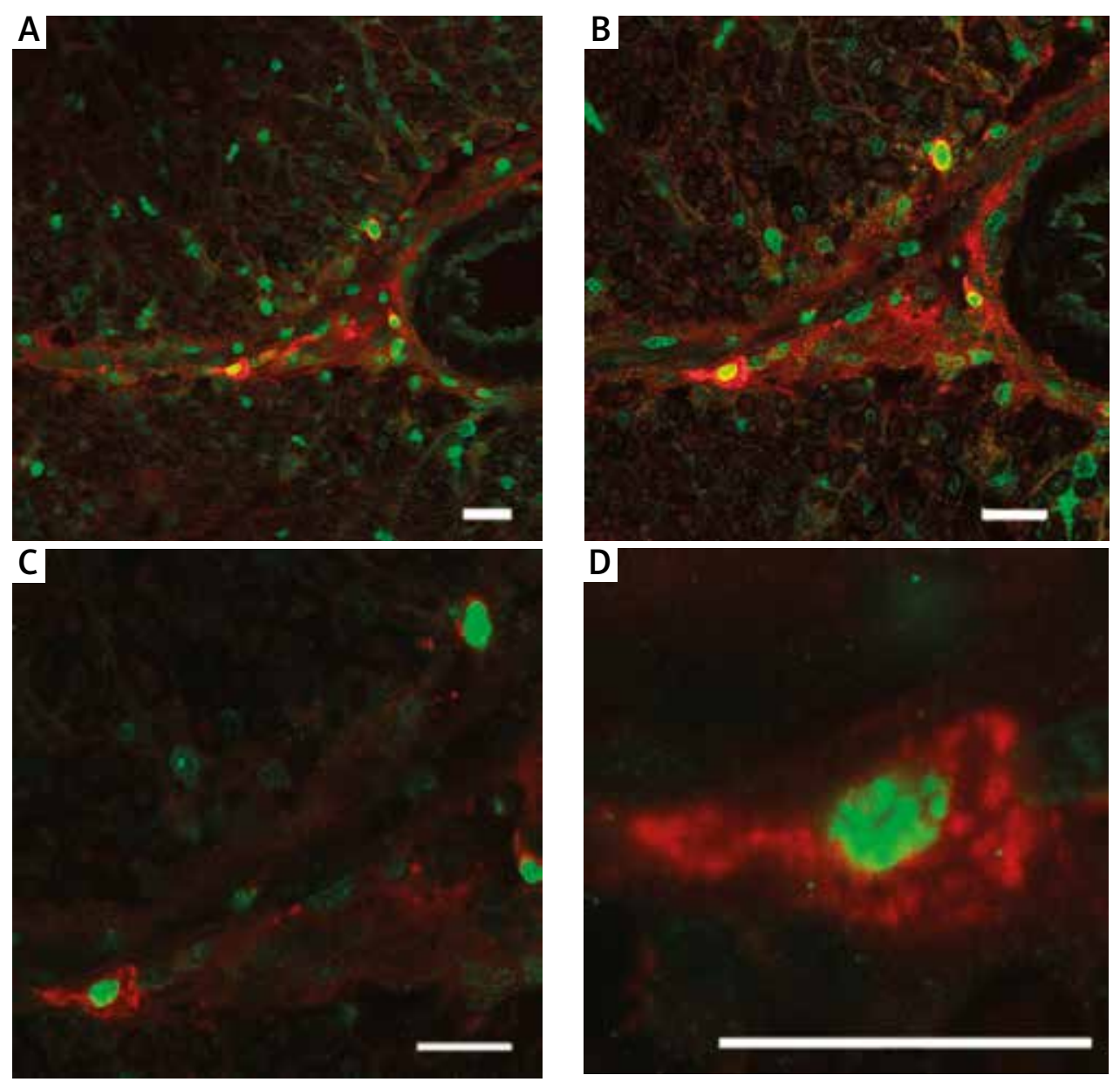

Fig. 4. Double fluorescence staining showing immunoreactivity for Ki67 and GFAP in the spinal cords of EAE animals. Bright grey (green in a colour photograph) represents Ki67 positivity, and darker grey (red in a colour photograph) represents GFAP positivity. The photomicrographs were taken at magnification 630x. Scale bar corresponds to $20 \mu \mathrm{m}$.

Fluorescent immunoreactivities of Ki67 with GFAP and magnified photomicrograph in the spinal cord and the evaluations of obtained data are presented in Figures 3, 4 and 5.

Elevated Ki67 expression in the EAE group was associated with elevated GFAP, compared to controls (Fig. 5A, 5B, $p<0.05$ ). Both therapeutic protocols (EAE + iTBS and EAE + CTBS) induced decreased Ki67 and GFAP immunoreactivity, compared to the EAE group $(p<0.05)$. In these groups, both coefficients of correlations between Ki67 and GFAP were not so prominent (Fig. 5C, D), but Manders coefficient indicates a high degree of Ki67 and GFAP molecules colocalization. In the healthy animals that were treated with iTBS/cTBS, a low level of both M1 (Ki67) and M2 (GFAP) coefficients of colocalization had been found.
Double staining of BDNF with Iba 1 and data analyses of the spinal cord tissue are shown in Figures 6 and 7.

Contrary to elevated BDNF expression in the CFA group, it was decreased in EAE animals, compared to controls (Fig. 7A, $p<0.05$ ). In addition, BDNF expression increased in iTBS-treated EAE animals compared to controls $(p<0.05)$, and in EAE + iTBS and $\mathrm{EAE}+$ cTBS, compared to the EAE group $(p<0.05)$. Immunoreactivity of Iba 1 decreased in EAE, EAE + CTBS and cTBS animals, compared to controls (Fig. $7 \mathrm{~B}, p<0.05)$. Treatment with iTBS in EAE animals induced elevation of Iba 1 expression, compared to controls, as well as compared to the EAE group $(p<0.05)$. The Pearson's coefficients between BDNF and Iba 1, although at a low level, indicates 

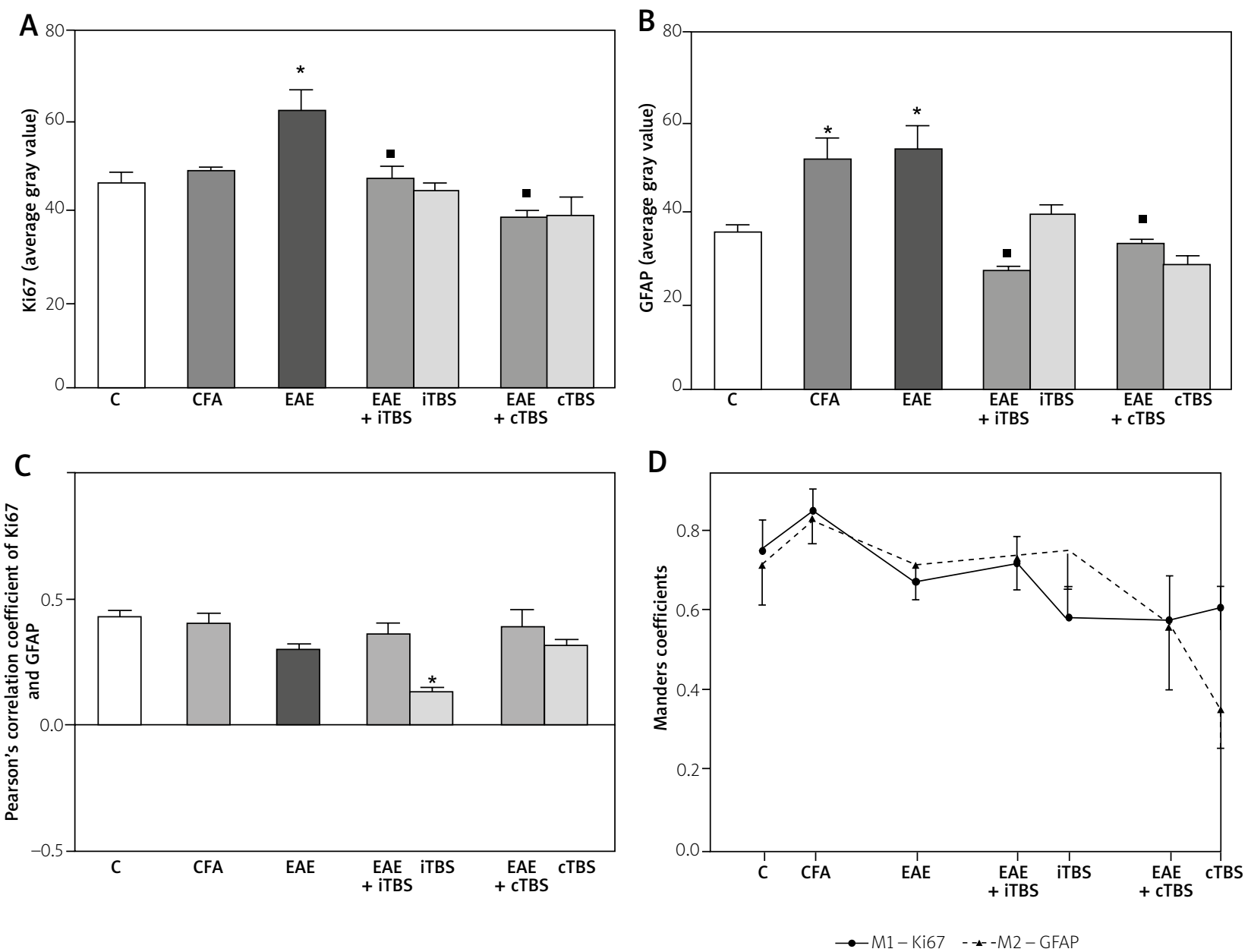

Fig. 5. Effects of iTBS and cTBS on Ki67 (A) and GFAP (B) expression in the spinal cord. Results were expressed as average grey value \pm SD $(n=3)$. Changes of Pearson's correlation coefficient $(C)$ and Manders coefficients (D): M1 (Ki67) and M2 (GFAP). Values are presented as means \pm SD. Differences were considered statistically significant for ${ }^{*} p<0.05$ compared to controls and $p<0.05$ compared to the EAE group.

increased values in all experimental groups (except CFA) compared to controls (Fig. 7C, $p<0.05$ ).

Fluorescent immunoreactivities of Ki67 with Iba 1 and the estimated values are shown in Figures 8 and 9 .

Expression of Ki67 together with Iba 1 is increased in EAE, compared to controls ( $p<0.05$ ), while iTBS treatment in EAE animals induced lower immunoreactivity of both molecules, compared to the EAE group (Fig. 9A, B, $p<0.05$ respectively). Increased Ki67 in EAE + cTBS ( $p<0.05)$ was not followed by changed Iba 1 expression. The coefficients of correlations and colocalizations between Ki67 and Iba 1 were not significant (Fig. 9C, D).

Double staining of BDNF and GABA and the confocal assessment of results are shown in Figures 10 and 11.
Decreased BDNF and increased GABA was noticed in the EAE group, compared to controls (Fig. 11A, B, $p<0.05$ respectively). Both therapeutic protocols (iTBS or (TBS) induced opposite changes - increased BDNF and reduced GABA expression in EAE animals, compared to the EAE group $(p<0.05)$. Expression of GABA in cTBS-treated healthy animals increased compared to controls $(p<0.05)$. Besides the low level of correlation, Manders coefficient of colocalization indicates divergent colocalization in the control group which is consistent only with EAE + iTBS group (Fig. 11C, D). Except for these two groups, all other groups showed a high level of BDNF and GABA colocalization. The statistical significance was obtained only for $M 2(G A B A)$ in $E A E+$ cTBS compared to the EAE group of animals $(p<0.05)$. 


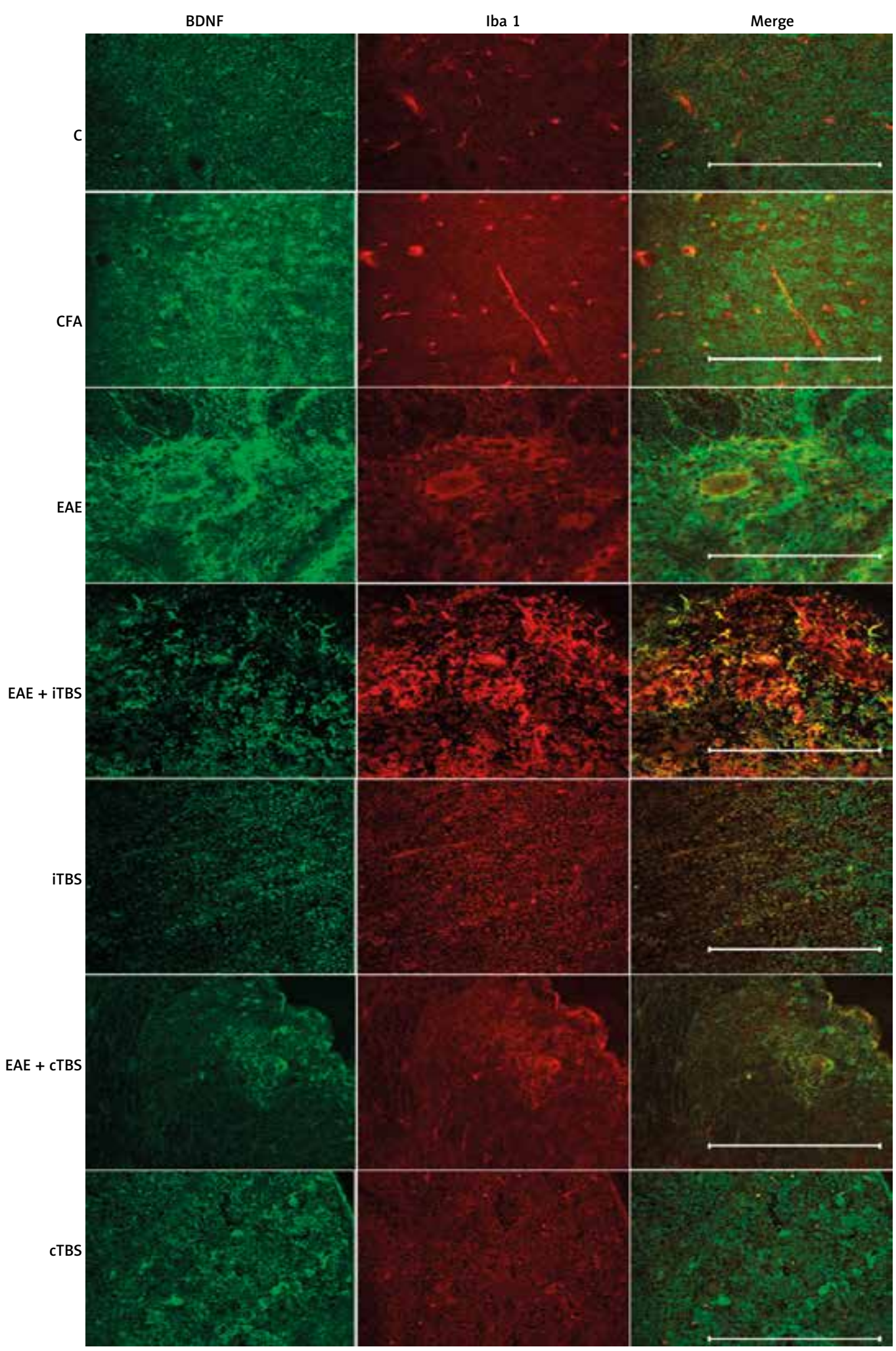

Fig. 6. Double fluorescence staining showing immunoreactivity for BDNF and lba 1 in rats' spinal cords. Bright grey (green in a colour photograph) represents BDNF positivity, and darker grey (red in a colour photograph) represents Iba 1 positivity. C - control group, EAE - rats (EAE) with experimental autoimmune encephalomyelitis, CFA - rats treated with complete Freund's adjuvant, iTBS treatment on EAE animals (EAE + iTBS) or on healthy animals (iTBS) and cTBS treatment on EAE animals (EAE + CTBS) or on healthy animals (cTBS). The photomicrographs were taken at magnification 400x. Scale bar corresponds to $200 \mu \mathrm{m}$. 

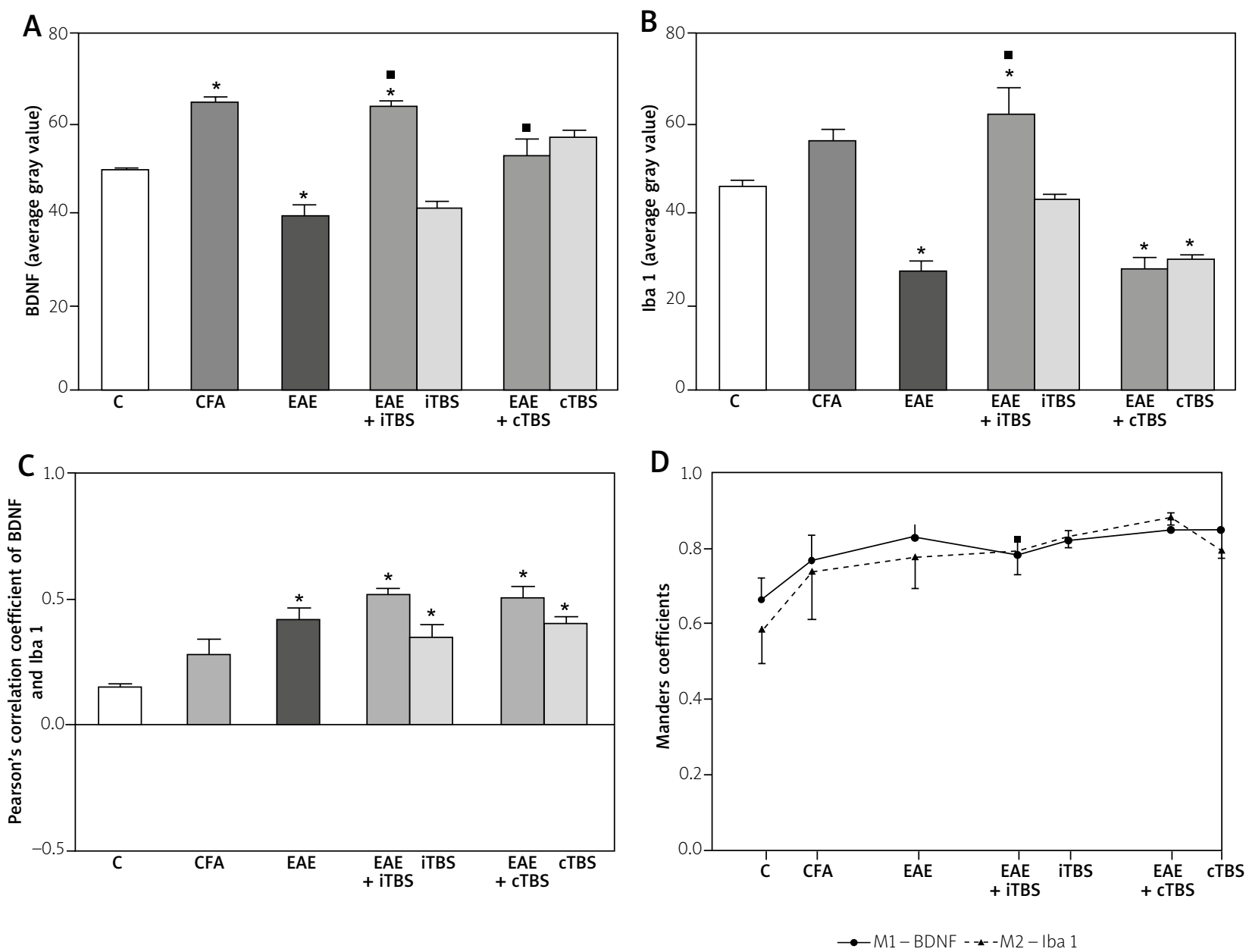

Fig. 7. Effects of iTBS and CTBS on BDNF (A) and Iba 1 (B) expression in the spinal cord. Results were expressed as average grey value \pm SD $(n=3)$. Changes of Pearson's correlation coefficient $(C)$ and Manders coefficients (D): M1 (BDNF) and M2 (Iba 1). Values are presented as means \pm SD. Differences were considered statistically significant for ${ }^{*} p<0.05$ compared to controls and $p<0.05$ compared to the EAE group.

\section{Discussion}

Decreased expression of BDNF in rats' spinal cord tissue of EAE animals in the phase of remission is consistent with previously published reports of early-term changes in EAE development (Figs. 2 and 7) [35]. Previously known as dorsal root ganglion-derived, BDNF is recognized to have functions in enhancements of neuronal survival following CNS damage: neurogenesis, the migration and differentiation of neurons, the growth of dendrites and axons, and synapse formation [5]. It had been shown that peripheral chronic BDNF administration in mice enhanced neuron survival [38]. Initially, mRNA BDNF transcripts are translated as a precursor protein,
proBDNF, which may be secreted into the synaptic cleft [43]. There, BDNF is cleaved by metalloproteinases and/or plasmin, giving rise to mature BDNF and the BDNF propeptide [22,32]. Then, BDNF is sorted into large dense core vesicles to be released from the regulated secretory pathway [29]. The decreased expression of BDNF in the remissive phase of EAE could be due to trafficking of BDNF to lysosomal degradation [8]. Also, decreased BDNF expression was associated with increased Ki67 and GFAP, the important marks of astrogliosis, which seems to be prolonged to the EAE remission (24 dpi). Assuming that microglia are not diffusely distributed in spinal cord parenchyma, decreased lba 1 together with 




Fig. 8. Double fluorescence staining showing immunoreactivity for Ki67 and Iba 1 in rats' spinal cords. Bright grey (green in a colour photograph) represents Ki67 positivity, and darker grey (red in a colour photograph) represents Iba 1 positivity. C - control group, EAE - rats (EAE) with experimental autoimmune encephalomyelitis, CFA - rats treated with complete Freund's adjuvant, iTBS treatment on EAE animals (EAE + iTBS) or on healthy animals (iTBS) and cTBS treatment on EAE animals (EAE + cTBS) or on healthy animals (cTBS). The photomicrographs were taken at magnification 400x. Scale bar corresponds to $200 \mu \mathrm{m}$. 

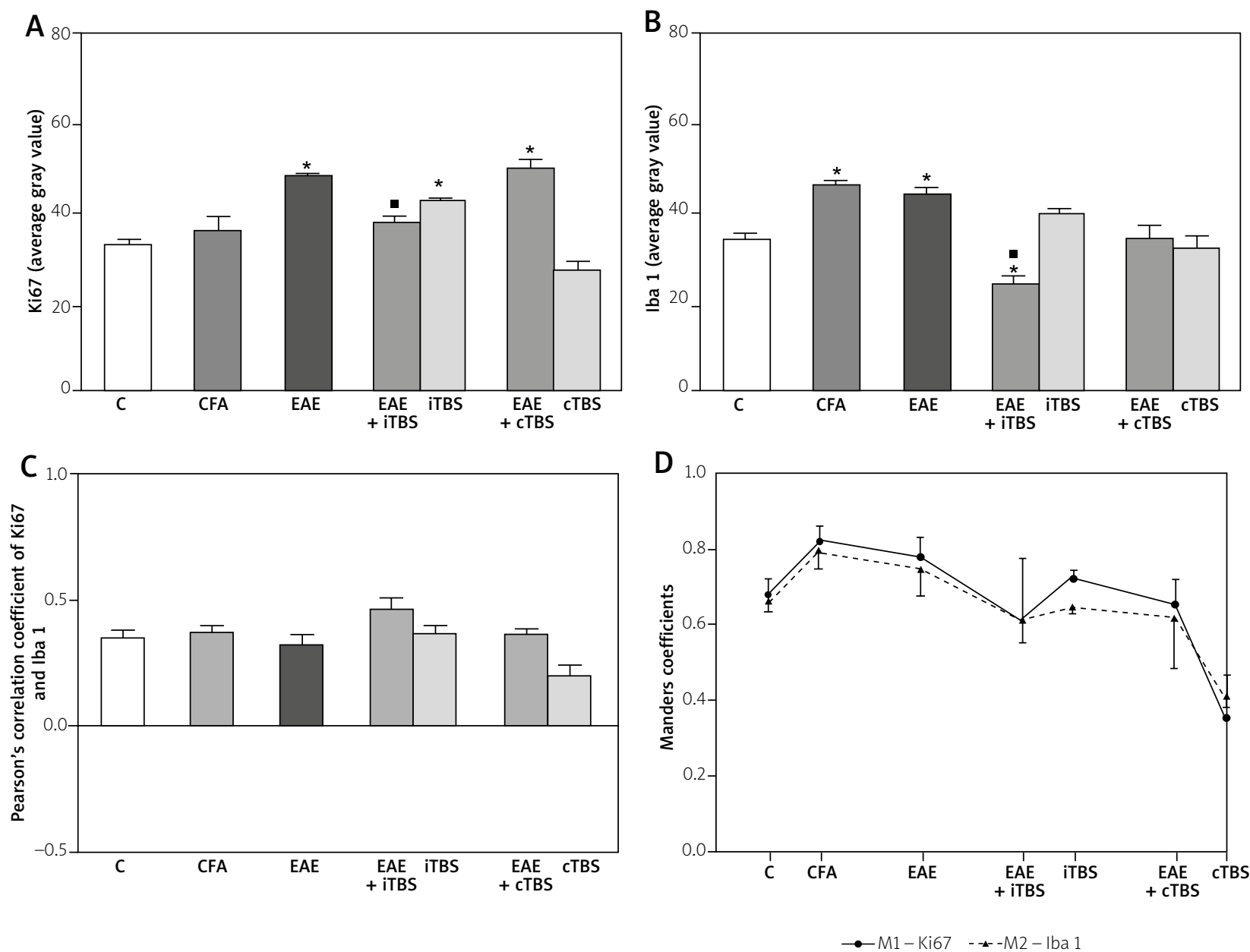

Fig. 9. Effects of iTBS and cTBS on Ki67 (A) and Iba 1 (B) expression in the spinal cords. Results were expressed as average grey value \pm SD $(n=3)$. Changes of Pearson's correlation coefficient $(C)$ and Manders coefficients (D): M1 (Ki67) and M2 (Iba 1). Values are presented as means \pm SD. Differences were considered statistically significant for ${ }^{*} p<0.05$ compared to controls and ${ }^{\|} p<0.05$ compared to the EAE group.

BDNF (Fig. 7) contrary to increased Iba 1 with Ki67 (Fig. 9) expression indicates clustered microglia in the resolution phase of EAE. Colocalizations of these molecules in EAE animals did not reveal any difference, compared to control animals. At the same time, GABA expression increased in spinal cord tissue sections, indicating higher GABA metabolic turnover, probably induced glutamic acid decarboxylase (GAD) activity in astrocytes, or prominent activity of GABAergic neurons (Fig. 11). Inflammation in EAE is related with calcium overload of neurons and manifested process of excitotoxicity in which the release of glutamate is a crucial manifestation. Such changes are the main events during the relapse phase of EAE, accordingly, increased GABA expression could indicate suppressed excitotoxicity and promoted inhibitory neuronal pathways [12].

Therapeutic procedures of both TBS induced important changes in investigated molecules expressions, such as BDNF elevation. Application of iTBS induced increasing of BDNF, compared to EAE animals, together with normalized GFAP and Ki67 (Figs. 2 and 5). Resting microgliosis with lower Ki67 in EAE + iTBS indicates clustered microglia with less mitotic activity. This is in accordance with large Manders coefficient of colocalization for BDNF and Iba 1 (Fig. 7D). It has been shown that BDNF support late hippocampal LTP through a TrkB-dependent mechanism. Panja et al. showed that LTP consolidation in the dentate gyrus is mediated on a translational level by sustained BDNF-TrkB signalling to 




Fig. 10. Double fluorescence staining showing immunoreactivity for BDNF and GABA in rats' spinal cords. Darker grey (red in a colour photograph) represents BDNF positivity, and bright grey (green in a colour photograph) represents GABA positivity. C - control group, EAE - rats (EAE) with experimental autoimmune encephalomyelitis, CFA - rats treated with complete Freund's adjuvant, iTBS treatment on EAE animals (EAE + iTBS) or on healthy animals (iTBS) and cTBS treatment on EAE animals (EAE + CTBS) or on healthy animals (cTBS). The photomicrographs were taken at magnification 400x. Scale bar corresponds to $200 \mu \mathrm{m}$. 

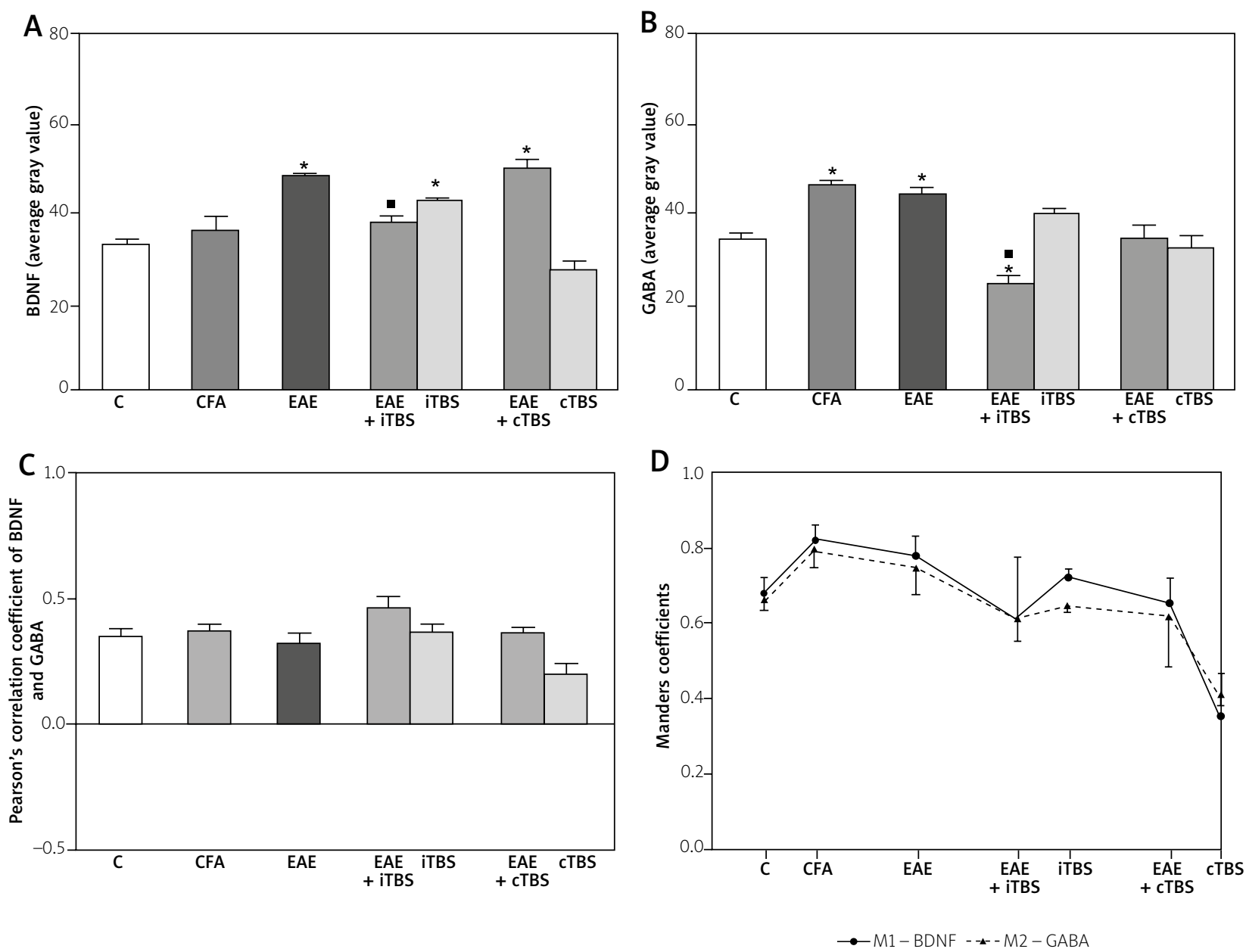

Fig. 11. Effects of iTBS and CTBS on BDNF (A) and GABA (B) expression in the spinal cords. Results were expressed as average grey value \pm SD $(n=3)$. Changes of Pearson's correlation coefficient $(C)$ and Manders coefficients (D): M1 (BDNF) and M2 (GABA). Values are presented as means \pm SD. Differences were considered statistically significant for ${ }^{*} p<0.05$ compared to controls and $p<0.05$ compared to the EAE group.

MAP-kinase-interacting kinase [33]. Advertising of LTP induced by iTBS is expected to trigger glutamatergic neurotransmission, which is crucial in the spinal cord. Promotion of BDNF neurotrophic effects post iTBS in EAE may support the survival of neurons in the dorsal horns as well as in the dorsal root ganglion [15]. It had been shown that patients with amyotrophic lateral sclerosis exhibit reduced serum levels of BDNF after the application of low-frequency rTMS to the motor cortex while the high-frequency stimulation increases BDNF levels in the blood plasma of patients with depression $[3,44]$. Our results are consistent in beneficial effects of both applied TMS protocols expressed as elevated BDNF in lumbar ventral horn regions of rats spinal cord.
BDNF binds TrkB with a high affinity [40]. Phosphorylation of residues at the cytoplasmic autoregulatory loop of the catalytic domain is considered a critical step in TrkB activation, followed by phosphorylation of other tyrosine residues triggering the MAPK/AKT pathways and activation of the signalling pathway through phospholipase $C \gamma[16,37]$. It had been shown that BDNF-induced TrkB activation is able to promote the survival of cultured embryonic mesencephalic dopaminergic neurons [18,21], cholinergic neurons in the basal forebrain [2], retinal ganglion cells [19], and also cortical embryonic neurons [36].

There are two primary rTMS treatment regimes: low-frequency rTMS, which is defined by stimulation 
at frequencies lower than $1 \mathrm{~Hz}$, and high-frequency rTMS, which is defined by stimulation at frequencies higher than $5 \mathrm{~Hz}$. Low-frequency rTMS reduces neuronal excitability, where a high-frequency rTMS increases cortical excitability [24]. In hippocampal cell cultures, low-intensity TMS $(1.14 \mathrm{~T}, 1 \mathrm{~Hz})$ results in dendritic sprouting (axon growth) and growth and increases the density of synaptic contacts. By contrast, high-intensity stimulation (1.55 T, $1 \mathrm{~Hz}$ ) has devastating effects that result in decreased numbers of dendrites and axons, the presence of neuronal lesions, and a diminished number of synapses. Both results are associated with the BDNF-TrkB signalling system [23].

Long-lasting positive effects of rTMS seem to act at the single neuron, neural network (mutual excitation and inhibition of cerebral regions), synaptic, and/or molecular (gene expression, enzyme activity, and neuromodulation) levels. Numerous studies have unambiguously demonstrated that TMS stimulate and induce gene expression and enhance the production of a number of signal molecules [39]. In an animal model of spinal cord injury, under the influence of TMS, astrocytes increased the ability to migrate to a zone of the lesion, which might be due to the activation of MEK1,2/ ERK mitotic pathways, and enhanced expressions of several genes [9].

In our experiment, changes in molecular expressions indicate beneficial effects of TBS/increased BDNF together with lower GFAP and Ki67, as well as GABA depletion. At the moment we could speculate influenced GAD activity in astrocytes, and/or modulated activity of GABAergic neurons. Effects of both TBS protocols suggest the TBS as potential therapeutic protocols in multiple sclerosis.

\section{Acknowledgements}

This work was supported by the University of Defence, Project No. MFVMA/04/19-21 and Ministry of Education and Science Republic of Serbia (grant number 41018).

\section{Disclosure}

The authors report no conflict of interest.

\section{References}

1. Agnati LF, Fuxe K, Torvinen M, Genedani S, Franco R, Watson S, Nussdorfer GG, Leo G, Guidolin D. New methods to evaluate colocalization of fluorophores in immunocytochemical preparations asexemplified by a study on A2A and D2 receptors in Chinese hamster ovary cells. J Histochem Cytochem 2005; 53: 941-953.
2. Alderson RF, Curtis R, Alterman AL, Lindsay RM, DiStefano PS. Truncated TrkB mediates the endocytosis and release of BDNF and neurotrophin-4/5 by rat astrocytes and schwann cells in vitro. Brain Res 2000; 871: 210-222.

3. Angelucci F, Oliviero A, Pilato F, Saturno E, Dileone M, Versace V, Musumeci G, Batocchi AP, Tonali PA, Di Lazzaro V. Transcranial magnetic stimulation and BDNF plasma levels in amyotrophic lateral sclerosis. Neuroreport 2004; 15: 717-720.

4. Aydin-Abidin S, Trippe J, Funke K, Eysel UT, Benali A. High- and lowfrequency repetitive transcranial magnetic stimulation differentially activates c-Fos and zif268 protein expression in the rat brain. Exp Brain Res 2008; 188: 249e261.

5. Baquet ZC, Gorski Jessica A, Jones KR. Early striatal dendrite deficits followed by neuron loss with advancedage in the absence of anterograde cortical brain derived neurotrophic factor. J Neurosci 2004; 24: 4250-4258.

6. Calabrese M, Filippi M, Gallo P. Cortical lesions in multiple sclerosis. Nat Rev Neurol 2010; 6: 438-444.

7. Dalmau I, Vela JM, González B, Finsen B, Castellano B. Dynamics of microglia in the developing rat brain. J Comp Neurol 2003; 458: 144-157.

8. Evans SF, Irmady K, Ostrow K, Kim T, Nykjaer A, Saftig P, Blobel C, Hempstead BL. Neuronal brain-derived neurotrophic factor is synthesized in excess, with levels regulated by sortilin-mediated trafficking and lysosomal degradation. J Biol Chem 2011; 286: 29556-29567.

9. Fang ZY, Li Z, Xiong L, Huang J, Huang XL. Magnetic stimulation influences injury-induced migration of white matter astrocytes. Electromagn Biol Med 2010; 29: 113-121.

10. Funke K, Benali A. Modulation of cortical inhibition by rTMS - findings obtained from animal models. J Physiol 2011; 589 : $4423 e 4435$.

11. Gedge L, Beaudoin A, Lazowski L, du Toit R, Jokic R, Milev R. Effects of electroconvulsive therapy and repetitive transcranial magnetic stimulation on serum brain-derived neurotrophic factor levels in patients with depression. Front Psychiatry 2012; 3: 12.

12. Gilani AA, Dash RP, Jivrajani MN, Thakur SK, Nivsarkar M. Evaluation of GABAergic transmission modulation as a novel functional target for management of multiple sclerosis: exploring inhibitory effect of GABA on glutamate-mediated excitotoxicity. Adv Pharmacol Sci 2014; 2014: 632376.

13. Gonzalez-Burgos G, Hashimoto T, Lewis DA. Inhibition and timing in cortical neural circuits. Am J Psychiatry 2007; 164: 12.

14. Hammer LA, Zagon IS, McLaughlin PJ. Improved clinical behavior of established relapsing-remitting experimental autoimmune encephalomyelitis following treatment with endogenous opioids: Implications for the treatment of multiple sclerosis. Brain Res Bull 2015; 112: 42-51.

15. Hofer MM, Barde YA. Brain-derived neurotrophic factor prevents neuronal death in vivo. Nature 1988; 331: 261-262.

16. Huang EJ, Wilkinson GA, Fariñas I, Backus C, Zang K, Wong SL, Reichardt LF. Expression of Trk receptors in the developing mouse trigeminal ganglion: in vivo evidence for NT-3 activation of TrkA and TrkB in addition to TrkC. Development 1999; 126: 2191-2203.

17. Huang YZ, Chen RS, Rothwell JC, Wen HY. The after-effect of human theta burst stimulation is NMDA receptor dependent. Clin Neurophysiol 2007; 118: 1028-1032. 
18. Hyman C, Juhasz M, Jackson C, Wright P, Ip NY, Lindsay RM. Overlapping and distinct actions of the neurotrophins BDNF, NT-3, and NT-4/5 on cultured dopaminergic and GABAergic neurons of the ventral mesencephalon. J Neurosci 1994; 14: 335-347.

19. Johnson RA, Lam M, Punzo AM, Li H, Lin BR, Ye K, Mitchell GS, Chang Q. 7,8-dihydroxyflavone exhibits therapeutic efficacy in a mouse model of Rett syndrome. J Appl Physiol (1985) 2012; 112: 704-710.

20. Kennedy MK, Torrance DS, Picha KS, Mohler KM. Analysis of cytokine mRNA expression in the central nervous system of mice with experimental autoimmune encephalomyelitis reveals that IL-10 mRNA expression correlates with recovery. I Immunol 1992; 149: 2496-2505

21. Knusel B, Gao H, Okazaki T, Yoshida T, Mori N, Hefti F, Kaplan DR. Ligand-induced down-regulation of Trk messenger RNA, protein and tyrosine phosphorylation in rat cortical neurons. Neuroscience 1997; 78: 851-862.

22. Lee R, Kermani P, Teng KK, Hempstead BL. Regulation of cell survival by secreted proneurotrophins. Science 2001; 294: 1945-1948.

23. Ma J, Zhang Z, Su Y, Kang L, Geng D, Wang Y, Luan F, Wang M, Cu H. Magnetic stimulation modulates structural synaptic plasticity and regulates BDNF-TrkB signal pathway in cultured hippocampal neurons. Neurochem Int 2013; 62: 84-91.

24. Maeda F, Keenan JP, Tormos JM, Topka H, Pascual-Leone A. Interindividual variability of the modulatory effects of repetitive transcranial magnetic stimulation on cortical excitability. Exp Brain Res 2000; 133: 425-430.

25. Mancic B, Stevanovic I, Ilic TV, Djuric A, Stojanovic I, Milanovic S, Ninkovic M. Transcranial theta-burst stimulation alters GLT-1 and vGluT1 expression in rat cerebellar cortex. Neurochem Int 2016; 100: 120-127.

26. Manyam NV, Katz L, Hare TA, Gerber JC 3rd, Grossman MH. Levels of gamma-aminobutyric acid in cerebrospinal fluid in various neurologic disorders. Arch Neurol 1980; 37: 352-355.

27. Martins LF, Costa RO, Pedro JR, Aguiar P, Serra SC, Teixeira FG, Sousa N, Salgado AJ, Almeida RD. Mesenchymal stem cells secretome-induced axonal outgrowth is mediated by BDNF. Sci Rep 2017; 7: 4153

28. Massella A, D'Intino G, Fernández M, Sivilia S, Lorenzini L, Giatti S, Melcangi RC, Calzà L Giardino L Gender effect on neurodegeneration and myelin markers in an animal model for multiple sclerosis. BMC Neurosci 2012; 13: 12.

29. Matsuda N, Lu H, Fukata Y, Noritake J, Gao H, Mukherjee S, Nemoto T, Fukata M, Poo MM. Differential activity-dependent secretion of brain-derived neurotrophic factor from axon and dendrite. J Neurosci 2009; 29: 14185-14198.

30. Muhallab S, Lundberg C, Gielen AW, Lidman O, Svenningsson A, Piehl F, Ollson T. Differential expression neurotrophic factors and inflammatory cytokines by myelin basic protein-specific and other recruited T cells infiltrating the central nervous system during experimental autoimmune encephalomyelitis. Scand I Immunol 2002; 55: 264-273.

31. Musella A, Gentile A, Rizzo FR, De Vito F, Fresegna D, Bullitta S, Vanni V, Guadalupi L, Stampanoni Bassi M, Buttari F, Centonze D, Mandolesi G. Interplay between age and neuroinflammation in multiple sclerosis: Effects on motor and cognitive functions. Front Aging Neurosci 2018; 10: 238.
32. Pang PT, Lu B. Regulation of late-phase LTP and long-term memory in normal and aging hippocampus: role of secreted proteins tPA and BDNF. Ageing Res Rev 2004; 3: 407-430.

33. Panja D, Kenney JW, D'Andrea L, Zalfa F, Vedeler A, Wibrand K, Fukunaga R, Bagni C, Proud CG, Bramham CR. Two-stage translational control of dentate gyrus LTP consolidation is mediated by sustained BDNF-TrkB signaling to MNK. Cell Rep 2014; 9: 14301445.

34. Paparrigopoulos T, Ferentinos P, Kouzoupis A, Koutsis G, Papadimitriou GN. The neuropsychiatry of multiple sclerosis: focus on disorders of mood, affect and behaviour. Int Rev Psychiatry 2010; 22: 14-21.

35. Park H, Poo MM. Neurotrophin regulation of neural circuit development and function. Nat Rev Neurosci 2013; 14: 7-23.

36. Polleux F, Whitford KL, Dijkhuizen PA, Vitalis T, Ghosh A. Control of cortical interneuron migration by neurotrophins and PI3-kinase signaling. Development 2002; 129: 3147-3160.

37. Rantamäki T, Hendolin P, Kankaanpää A, Mijatovic J, Piepponen P, Domenici E, Chao MV, Männistö PT, Castrén E. Pharmacologically diverse antidepressants rapidly activate brain-derived neurotrophic factor receptorTrkB and induce phospholipase-Cgamma signaling pathways in mouse brain. Neuropsychopharmacol 2007; 32: 2152-2162.

38. Schmidt HD, Duman RS. Peripheral BDNF produces antidepressant-like effects in cellular and behavioral models. Neuropsychopharmacol 2011; 36: 550

39. Simis M, Adeyemo BO, Medeiros LF, Miraval F, Gagliardi RJ, Fregni F. Motor cortex-induced plasticity by noninvasive brain stimulation: a comparison between transcranial direct current stimulation and transcranialmagneticstimulation. Neuroreport 2013; 24: 973-975.

40. Soppet D, Escandon E, Maragos J, Middlemas DS, Reid SW, Blair J, Burton LE, Stanton BR, Kaplan DR, Hunter T, Nikolics K, Parada LF. The neurotrophic factors brain-derived neurotrophic factor and neurotrophin-3 are ligands for the trkB tyrosine kinase receptor. Cell 1991; 65: 895-903.

41. Sze SCW, Wong CKC, Yung KKL. Modulation of the gene expression of N-methyl-D-aspartate receptor NR2B subunit in the rat neostriatum by a single dose of specific antisense oligodeoxynucleotide. Neurochem Int 2001; 39: 319-327.

42. Wang HY, Crupi D, Liu J, Stucky A, Cruciata G, Di Rocco A. Repetitive transcranial magnetic stimulation enhances BDNF-TrkB signaling in both brain and lymphocyte. J Neurosci 2011; 31: 11044-11054.

43. Yang J, Siao CJ, Nagappan G, Marinic T, Jing D, McGrath K, Chen ZY, Mark W, Tessarollo L, Lee FS, Lu B, Hempstead BL. Neuronal release of proBDNF. Nat Neurosci 2009; 12: 113-115.

44. Yukimasa T, Yoshimura R, Tamagawa A, Uozumi T, Shinkai K, Ueda N. High-frequency repetitive transcranial magnetic stimulation improves refractory depression by influencing catecholamine and brain-derived neurotrophicfactors. Pharmacopsychiatry 2006; 39: 52-59.

45. Zhu W, Frost EE, Begum F, Vora P, Au K, Gong Y, MacNeil B, Pillai $P$, Namaka M. The role of dorsal root ganglia activation and brain-derived neurotrophic factor in multiple sclerosis. J Cell Mol Med 2012; 16: 1856-1865.

46. Zipoli V, Goretti B, Hakiki B, Siracusa G, Sorbi S, Portaccio E, Amato MP. Cognitive impairment predicts conversion to multiple sclerosis in clinically isolated syndromes. Mult Scler 2010; 16: 62-67. 